\title{
Creencias y actitudes de profesores y alumnos griegos de español ante las técnicas de corrección en la interacción oral: estudio comparativo intragrupos
}

\author{
Isabel SANTOS GARGALLO \\ Universidad Complutense de Madrid \\ isantos@edu.ucm.es \\ Angélica ALEXOPOULOU \\ Universidad Nacional y Kopodistríaca de Atenas \\ aalexop@spanll.uoa.gr
}

Recibido: abril 2014

Aceptado: julio 2014

RESUMEN: Este artículo explora las creencias o teorías implícitas de los alumnos y su actitud en relación con los errores que tienen lugar en la interacción oral y las técnicas de corrección que los profesores utilizan - qué, quién, cuándo y cómo corregir-; profundizar en el conocimiento de las creencias nos ayudará a entender su actitud y a orientar de forma adecuada el proceso de enseñanza-aprendizaje. Desarrollamos un estudio de caso seudolongitudinal de corte cuantitativo y adoptamos la encuesta de respuesta cerrada como instrumento para la recogida de datos.

Palabras clave: creencias, profesor, alumno, corrección de los errores, interacción oral, español como lengua extranjera (ELE).

Beliefs and Attitudes of Teachers and Greek Learners of Spanish towards Techniques of Correction in Oral Interaction:

An Intergroup Comparative Study

\begin{abstract}
This article examines learners' beliefs or implicit theories and their attitude in relation to errors which occur in oral interaction as well as the techniques of correction teachers use - what, whom, when and how to correct-; deepening our knowledge of their beliefs will help us understand their attitude and design an adequate method of teaching learning. We are conducting a pseudo-longitudinal quantitative case study, adopting a questionnaire of closed questions as an instrument to collect data.
\end{abstract}

Keywords: teachers and students' assumptions and beliefs, error correction, oral interaction, Spanish as a Foreign Language. 
Croyances et attitudes des professeurs et apprenants grecs d'espagnol confrontés aux techniques de correction dans l'interaction orale : une étude comparative intra-groupe

RÉSUMÉ: Cet article explore les croyances ou théories implicites des apprenants et leur attitude lorsqu'ils sont confrontés aux erreurs qui ont lieu dans l'interaction orale, aussi bien que les techniques de correction que les enseignants appliquent - que, qui, quand et comment corriger - ; approfondir notre connaissance de ces "croyances" peut, d'une part, nous aider à comprendre les attitudes des uns et des autres et, d'autre part, à orienter d'une façon adéquate le processus d'enseignement-apprentissage. Nous avons mené une étude quantitative pseudo longitudinale, en utilisant comme outil pour la collecte des données un questionnaire composé de questions fermées.

Mots-clés: croyances des enseignants et des apprenants, correction des erreurs, interaction orale, Espagnol comme langue étrangère.

SUMARIO: 1. Introducción. 2. Objetivos. 3. Metodología de la investigación. 4. Muestra. 4.1. Alumnos. 4.2. Profesores. 5. Análisis y discusión de los datos. 5.1. Alumnos y profesores están de acuerdo. 5.2. Alumnos y profesores no están de acuerdo. 5.3. Comparación de variables intragrupos: A2, B1, B2 y C1. 6. Conclusiones. 7. Bibliografía. Anexos.

\section{INTRODUCCIÓN}

En la presente investigación, a través de un estudio de caso seudolongitudinal de corte cuantitativo-, nos proponemos indagar en las creencias de profesores y alumnos griegos de E/LE y en su actitud en relación con los errores que tienen lugar en la interacción oral, con el propósito de descubrir qué importancia conceden los aprendientes a las técnicas de corrección que los profesores utilizan en el aula.

La amplia difusión del nuevo enfoque metodológico que surge alrededor de los años setenta y que propone el logro de una competencia comunicativa (MCRE, 2002) que permitirá al hablante no nativo actuar adecuadamente en cada situación comunicativa, ha situado al alumno en el centro del proceso de aprendizaje de una L2 convirtiéndolo en el protagonista indiscutible. Con este cambio de perspectiva se empiezan a priorizar opciones metodológicas que toman en cuenta las necesidades y las expectativas del aprendiente, sus diferentes formas de aprender, las estrategias que pone en marcha y sus experiencias previas con otras lenguas. Asimismo, se empiezan a considerar los factores individuales que intervienen a lo largo del proceso de aprendizaje/adquisición de una lengua extranjera, factores que en gran medida explican las diferencias en los resultados obtenidos. En este contexto, se revela la importancia de reflexionar sobre las creencias, las actitudes, las necesidades y las expectativas con las que los alumnos llegan a clase.

Las creencias o representaciones mentales son recursos cognitivos conformados por concepciones preestablecidas que posee el ser humano para organizar, 
describir e interpretar el mundo y las experiencias que lo rodean. Constituyen una herramineta para procesar la información y funcionan como una guía de actuación. Puchta $(2000,273)$ sostiene que "actúan como filtros muy fuertes de la realidad" y Van Dijk (1999, 43) afirma que "las personas estructuran, comprenden y experimentan el mundo (directamente o por medio de instrumentos) en términos de sus creencias". A pesar de no estar fundamentadas en ningún tipo de investigación son tenidas por verdaderas y se identifican con la realidad, puesto que "si creemos en algo, actuamos como si fuera verdad, a pesar de la realidad" (Puchta, 2000, 273).

Concepto un tanto impreciso, las "creencias" son un constructo difícil de delimitar por su fuerte carga de subjetividad. Pajares (1992) en su intento por despejar el ámbito conceptual ofrece veintiuna denominaciones distintas encontradas en la bibliografía. Con toda la dificultad que puede suponer definir un concepto por naturaleza poliédrico, los estudiosos del tema coinciden en una serie de características resumidas en la definición que propone Ramos Méndez (2007, 21):

... ideas relativamente estables que tiene un individuo sobre un tema determinado, forjadas a través de su experiencia personal bajo la influencia de un proceso de construcción social, agrupadas en redes o sistemas, de cuya veracidad está convencido y que actúan como un filtro a través del cual percibe e interpreta el mundo que lo rodea, tomando sus decisiones de acuerdo con ello.

En el ámbito del aprendizaje de lenguas extranjeras, las creencias juegan un papel fundamental en el proceso: los aprendientes llegan al aula con ideas preestablecidas determinadas por sus creencias respecto a multitud de aspectos que se refieren a la naturaleza del lenguaje, a la propia lengua meta, a sus hablantes y a los países donde se habla, a la dificultad para aprenderla, al tratamiento del error, a lo que hay que aprender, cómo, con qué materiales y en cuanto tiempo. Puesto que muchas de estas creencias están ancladas en conocimientos incorrectos o, en el mejor de los casos, incompletos, muchas de ellas son discutibles e inducen a falsas decisiones. Los aprendientes, por lo tanto, llegan al aula con su propia forma de abordar el aprendizaje, con unos hábitos formados y, lo más importante, con una visión que tienen de ellos mismos. Como apunta Arnold (2006) "un alumno que tiene la creencia de que no puede aprender tiene razón, no puede... a menos que cambie esta creencia".

Todas estas creencias, fruto de su experiencia previa como aprendientes de lenguas, de la enseñanza que hayan experimentado y de los profesores que hayan tenido, pero también de cómo esté considerado el aprendizaje de lenguas en su entorno cultural y educativo, así como del papel que les otorgue dicho entorno a ellos como alumnos (Ramos Méndez, 2007), son susceptibles de desempeñar un papel muy importante en el proceso de apropiación de una L2. Esto se hace patente 
si consideramos su influencia en la conducta de los alumnos: afectan a su manera de aprender, actúan como un filtro en su percepción de lo que pasa en el aula, determinan las estrategias de aprendizaje que ponen en práctica para que el proceso sea más efectivo; en una palabra, repercuten directamente en el rendimiento de los aprendientes. Por otro lado, la toma de conciencia por parte del alumno de sus propias representaciones fomenta la autonomía en el aprendizaje y el aprender a aprender, dos conceptos fundamentales en el planteamiento general del enfoque comunicativo y de la enseñanza orientada a la acción.

Las creencias, al ser un componente asociado al deseo de aprender, crean unas expectativas que pueden potenciar el aprendizaje cuando son favorables $\mathrm{u}$ obstaculizarlo cuando son desfavorables. Es pues evidente el papel que desempeñan los factores individuales, tanto afectivos (ansiedad, actitud, motivación etc.) como cognitivos (estilos de aprendizaje, inteligencia, aptitud etc.). De hecho, los factores individuales que influyen en el aprendizaje constituyen un marco para el estudio de las creencias; la ansiedad del alumno ante el error es un ejemplo significativo.

Pero, no se trata solamente de las creencias de los alumnos; en más de una ocasión se ha puesto de manifiesto el impacto que tiene el pensamiento del profesorado en el proceso educativo; los profesores, como los alumnos, llegan al aula con su propio bagaje de creencias y representaciones mentales formadas a partir de sus propias experiencias como alumnos de lenguas, de los rasgos de su personalidad, así como de su formación profesional. Su actuación docente depende no solamente de sus conocimientos, sino también de una red compleja de teorías personales, concepciones y creencias, que se evidencian en su forma de enseñar. Según Williams y Burden $(1999,65)$, "las creencias de los profesores respecto a lo que es la enseñanza afecta a todo lo que hacen en el aula, tanto si estas creencias son implícitas como explícitas". De ahí la importancia de estudiar las relaciones existentes entre el sistema de creencias y la actividad docente.

En cuanto a la conformación de las creencias de los profesores en relación con los errores y su corrección no cabe duda que se trata de un condicionante ineludible de la actitud de los alumnos (Mekassimove, 2009). El estudio de Díaz Martínez (1998) aborda, desde la perspectiva cualitativa y el diario introspectivo como técnica- la relación entre los factores afectivos y la actitud ante los errores; y pone de manifiesto que la falta de afectividad y empatía por parte del profesor ante la corrección de los errores en la producción oral -especialmente en la pronunciaciónproduce en el aprendiente falta de receptividad, desmotivación e, incluso, rebeldía. Insiste y destaca que lo afectivo puede ser incluso más determinante para el aprendizaje que los propios contenidos. Sin embargo, los resultados no permiten la generalización por tratarse de una sola informante y ésta coincidir con la investigadora. En esta misma línea, Fernández de Amo (2008) desarrolla el concepto de error afectivo, para referirse a aquellos errores que no responden a la falta de conocimiento o competencia sino a timidez, inhibición o miedo a 
exponerse (estos podrían relacionarse con la categoría faltas de Corder, 1967); también Roncel Vega (2008) se interesa en su estudio por la misma correlación analizando el autoconcepto, la motivación y la ansiedad en estudiantes eslavos de español.

Por último, cabe mencionar la importancia especial que concede a las creencias el Marco Común Europeo de Referencia para las lenguas (2002). Este documento contempla un primer grupo de competencias -las competencias generales- a las que el usuario de la lengua recurre para acciones de todo tipo, que a su vez están conformadas por cuatro tipos de saberes (saber, saber ser, saber hacer, saber aprender) que apelan a diferentes objetivos. Las creencias aparecen recogidas, junto con las actitudes y los valores, dentro de las competencias generales, como parte del conocimiento declarativo (saber) y, en concreto, del conocimiento sociocultural, y al mismo tiempo forman parte de la competencia existencial (saber ser), estrechamente relacionada con los rasgos de la personalidad del aprendiente.

Todas estas implicaciones revelan la importancia de profundizar en la investigación sobre el sistema de creencias con las que los alumnos llegan a la clase de ELE, dado que al determinar su forma de abordar el aprendizaje, ejercen una influencia central en la toma de decisiones de aprendientes y docentes. El mejor conocimiento del universo mental del estudiante no puede sino incidir positivamente en el proceso de aprendizaje.

El estudio de las creencias de los alumnos empieza a ser desarrollado de manera más sistemática por los investigadores a partir de la publicación del test BALLI (Beliefs About Language Learning Inventory) diseñado por Horwitz (1987), si bien los análisis de necesidades y los trabajos sobre estrategias de aprendizaje pueden ser considerados como estudios que abren esta línea de investigación, pues todos ellos se orientan a trazar el perfil del alumno con el fin de guiar el proceso de enseñanza-aprendizaje.

De tal manera, con este estudio empírico pretendemos comprobar si efectivamente hay una relación entre el pensamiento del profesor, su actuación en el aula y la actitud de los alumnos; y si, asimismo, la actitud de los alumnos modifica y condiciona la actuación del profesor. Nuestra investigación se centra en el papel de los errores, qué técnicas prefiere para su corrección en la interacción oral, qué quiere que le corrijan, quién quiere que le corrija, cuándo quiere que le corrijan y cómo quiere que le corrijan, entre otros aspectos.

Pasamos a explicitar los objetivos, la metodología y las características de la muestra de este estudio de caso, para después, llevar a cabo el análisis de los datos y la discusión de los mismos, atendiendo especialmente a la correlación entre las variables que tengan significación (sig.) según la prueba t de Student. 


\section{OBJETIVOS}

Esta investigación se propone como objetivo general averiguar cuáles son las creencias de profesores y alumnos en su relación con los errores en la interacción oral, y cómo el pensamiento condiciona su actitud y conducta ante las técnicas de corrección; dicho objetivo se concreta en los siguientes específicos -profesores vs.alumnos-:

1. Consideración positiva/negativa ante los errores.

2. Preferencias en relación con quién corrige.

3. Preferencias en relación con qué corregir.

4. Preferencias en relación con cuándo corregir.

5. Preferencias en relación con cómo corregir.

\section{METODOLOGÍA DE LA INVESTIGACIÓN}

Los estudios sobre las teorías implícitas y las creencias del profesor y del alumno pueden ser abordados desde cualquiera de los dos paradigmas científicos cuantitativo y cualitativo- o combinar ambos, ya que, de acuerdo con Nunan (1992), creemos que en lingüística aplicada y adquisición de segundas lenguas (ASL), la adopción de métodos y técnicas adscritas a ambos o a cualquiera de los dos puede resultar provechosa y proporcionar una visión más completa sobre cuestiones en las que el informante y proveedor de los datos es un sujeto complejo y los fenómenos que se investigan, poliédricos: estos dos paradigmas no resultan excluyentes sino complementarios, no existe cisma entre ellos, tal y como afirman Larsen Freeman y Long $(1994,32)$, consideramos que "tenemos mucho que ganar aproximándonos al estudio de la ASL combinando las ventajas de los paradigmas cuantitativo y cualitativo. En vez de considerarlos como paradigmas opuestos, entendemos que son complementarios, y con ello implicamos que no hay que optar necesariamente por uno u otro".

Algunas de las investigaciones sobre el pensamiento del profesor $\mathrm{y} / \mathrm{o}$ del alumno han sido abordadas desde la perspectiva cualitativa, priorizándose en ellas los aspectos exploratorios; no obstante, en este estudio, hemos decidido acogernos al paradigma cuantitativo - positivista, objetivo, medible, verificable y orientado hacia los resultados- con el fin de abordar una muestra más amplia y aumentar, así, la capacidad de generalización y la posible contrastación del estudio, en lo que a los alumnos se refiere.

En el marco del paradigma cuantitativo, adoptamos el estudio de caso como método que "describe el comportamiento y la actuación lingüística de un número de hablantes a fin de generalizar las conclusiones al total de la población" (Yin, 1984, 14), o, al menos, a un porcentaje de la población. Un método empírico, selectivo y descriptivo que estudia un fenómeno -en nuestro caso, las creencias de los alumnos y de los profesores-, dentro de un contexto real de la vida cotidiana. 
Además, se trata de un estudio de carácter seudolongitudinal, ya que la muestra está constituida por alumnos de cuatro niveles de referencia consecutivos (A2, B1, $\mathrm{B} 2$ y $\mathrm{C} 1$ ), pero los datos han sido tomados de forma simultánea durante el mismo año académico.

En cuanto al instrumento adoptado para la compilación de los datos, hemos elegido el cuestionario de respuesta cerrada, que tal y como apuntan Richards y Lockhart $(1998,19)$ resulta "útil para recopilar información sobre las dimensiones afectivas de la enseñanza y el aprendizaje, tales como puntos de vista, actitudes, motivación y preferencias". El cuestionario para los profesores consta de 38 variables: las diez primeras establecen el perfil del informante y las 28 siguientes analizan su actitud ante los errores y la corrección de los mismos mediante enunciados o ítems tipo Likert; por otro lado, el cuestionario para los alumnos consta de 34 variables: las 8 primeras establecen el perfil del informante, y las 26 restantes, abordan las creencias y actitudes de los alumnos ante los errores y las técnicas empleadas en su corrección en la interacción oral. En la segunda parte de cada uno de los cuestionarios -28 ítems para profesores y 26, para alumnos-, el informante debe expresar su grado de acuerdo-desacuerdo en relación con los enunciados en una escala de 1 a 5 con los siguientes valores: (1) Estoy muy en desacuerdo; (2) Estoy en desacuerdo; (3) Ni de acuerdo ni en desacuerdo; (4) Estoy de acuerdo; y (5) Estoy muy de acuerdo (adaptado de Chaparro, 2008).

Para determinar el grado de acuerdo entre las creencias de los alumnos y las de los profesores, se aplica la prueba t del paquete estadístico SPSS que calcula la media entre la puntuación de cada uno de los grupos de informantes y establece los ítems en los que el contraste resulta significativo (sig.).

\section{MUESTRA}

La muestra, de tipo incidental, está constituida por 86 sujetos: de ellos, 81 son alumnos y 5 profesores, tal y como se refleja en la tabla 1 :

Tabla 1. Distribución de la muestra

\begin{tabular}{|l|c|c|c|}
\hline & $\begin{array}{c}\text { Centro de } \\
\text { Idiomas } \\
\text { Modernos de la } \\
\text { Universidad de } \\
\text { Atenas }\end{array}$ & $\begin{array}{c}\text { Universidad } \\
\text { Nacional y } \\
\text { Kapodistríaca de } \\
\text { Atenas }\end{array}$ & Total \\
\hline ALUMNOS & $\mathbf{6 8}$ & $\mathbf{1 3}$ & $\mathbf{8 1}$ \\
\hline PROFESORES & $\mathbf{4}$ & $\mathbf{1}$ & $\mathbf{5}$ \\
\hline TOTAL & $\mathbf{7 2}$ & $\mathbf{1 4}$ & $\mathbf{8 6}$ \\
\hline
\end{tabular}




\subsection{Alumnos}

La muestra para el estudio de las creencias de los alumnos está constituida por 81 informantes distribuidos en cuatro grupos correspondientes a los niveles de referencia $\mathrm{A} 2, \mathrm{~B} 1, \mathrm{~B} 2$ y C1, es decir, cada uno de los cuatro grupos está formado por 20 alumnos, a excepción del A2 (21 alumnos); pertenecen al Centro de Idiomas Modernos de la Universidad de Atenas en el caso de los niveles A2, B1 y B2, mientras que el $\mathrm{C} 1$ está constituido por 8 alumnos del mencionado Centro y 13 alumnos de la Universidad Nacional y Kapodistríaca de Atenas.

A continuación, resumimos en la tabla 2 los datos más significativos para establecer el perfil de los alumnos que forman parte de la muestra de este estudio:

Tabla 2. Datos significativos del perfil del alumno

\begin{tabular}{|c|c|c|c|}
\hline Variables & Válidos & Frecuencia & Porcentaje \\
\hline 1. Nivel & $\begin{array}{l}\text { A2 } \\
\text { B1 } \\
\text { B2 } \\
\text { C1 } \\
\text { Total }\end{array}$ & $\begin{array}{l}21 \\
20 \\
20 \\
20 \\
81\end{array}$ & $\begin{array}{c}25,9 \\
24,7 \\
24,7 \\
24,7 \\
100,0\end{array}$ \\
\hline 2. Edad & $\begin{array}{l}18-22 \\
23-30 \\
30-40 \\
40-62 \\
\text { Perdidos } \\
\text { Total }\end{array}$ & $\begin{array}{c}37 \\
15 \\
12 \\
4 \\
3 \\
81\end{array}$ & $\begin{array}{c}58,0 \\
18,5 \\
14,8 \\
4,8 \\
3,9 \\
100.0\end{array}$ \\
\hline 3. Sexo & $\begin{array}{l}\text { Mujer } \\
\text { Hombre } \\
\text { Total }\end{array}$ & $\begin{array}{l}61 \\
20 \\
81\end{array}$ & $\begin{array}{c}75,3 \\
24^{\prime} 7 \\
100,0\end{array}$ \\
\hline 4. $\begin{array}{l}\text { País de } \\
\text { origen }\end{array}$ & Grecia & 81 & 100,0 \\
\hline $\begin{array}{ll}\text { 5. } & \text { Lengua } \\
\text { materna (L1) }\end{array}$ & Griego & 81 & 100,0 \\
\hline $\begin{array}{ll}\text { 6. Otras } \\
\text { lenguas }\end{array}$ & $\begin{array}{l}\text { Inglés } \\
\text { Inglés y alemán } \\
\text { Inglés y francés } \\
\text { Total }\end{array}$ & $\begin{array}{l}25 \\
12 \\
32\end{array}$ & $\begin{array}{l}30,9 \\
14,8 \\
39,5\end{array}$ \\
\hline 7. Estudios & Superiores & 76 & 93,8 \\
\hline 8. Aprendo & Encontrar trabajo. & 28 & 34,6 \\
\hline
\end{tabular}




\begin{tabular}{|l|l|c|c|}
\hline español & Ayudar con los deberes del & 0 & 0,0 \\
para... & colegio. & 1 & 1,2 \\
& Participar en el barrio. & 29 & 35,8 \\
& Llenar un tiempo de ocio. & 34 & 42,0 \\
& Llegar a hablar muy bien. & & \\
& Aprender lo mínimo para & 28 & 34,6 \\
& comunicarme. & 25 & 30,9 \\
& Seguir estudiando algo. & 38 & 46,9 \\
& Entender a otras personas. & 44 & 54,3 \\
& Escuchar la radio, ver la & & \\
& televisión, etc. & \\
\hline
\end{tabular}

Los alumnos de la muestra son adultos con estudios superiores (96\%) y nacionalidad griega, por lo tanto, su lengua materna es el griego; no obstante un $85,2 \%$ sabe inglés como lengua extranjera; constituyen una mayoría de mujeres $(75,3 \%)$ y su edad oscila entre los 18 y los 62 años, sin embargo, el porcentaje más alto queda asignado a los alumnos que se encuentran entre los 18 y los 30 años (76,5\%), una media de edad que está en consonancia con los motivos por los que declaran estudiar español: encontrar trabajo (34,6\%), llenar un tiempo de ocio $(35,8 \%)$, llegar a hablar muy bien $(42,0 \%)$, aprender lo mínimo necesario para comunicarme $(30,9 \%)$, entender a otras personas $(46,9)$ y escuchar la radio, ver la televisión, etc $(54,3 \%)$ resultan los motivos más destacados y significativos (los informantes tuvieron la oportunidad se señalar varias de las opciones en este ítem).

\subsection{Profesores}

Los profesores incluidos en el estudio han sido un total de cinco: uno por cada grupo de alumnos (el correspondiente al grupo asignado) para los niveles A2, B1 y $\mathrm{B} 2$, y dos profesores para el nivel C1 (los correspondientes, también). En la tabla 3 presentamos las características más relevantes del perfil del grupo de profesores, ignorando aquellos datos que estadísticamente no resultan significativos:

Tabla 3. Datos significativos del perfil de los profesores

\begin{tabular}{|l|l|c|c|}
\hline \multicolumn{1}{|c|}{ Variables } & \multicolumn{1}{|c|}{ Válidos } & Frecuencia & Porcentaje \\
\hline \multirow{4}{*}{ 1. Edad } & 41 & 1 & 20,0 \\
& 42 & 2 & 40,0 \\
& 50 & 1 & 20,0 \\
& 52 & 1 & 20,0 \\
& Total & 5 & 100,0 \\
\hline
\end{tabular}




\begin{tabular}{|c|c|c|c|}
\hline 2. Sexo & $\begin{array}{l}\text { Mujer } \\
\text { Hombre } \\
\text { Total }\end{array}$ & $\begin{array}{l}4 \\
1 \\
5 \\
\end{array}$ & $\begin{array}{c}80,0 \\
20,0 \\
100,0 \\
\end{array}$ \\
\hline 3. País de origen & $\begin{array}{l}\text { España } \\
\text { Grecia } \\
\text { Total }\end{array}$ & $\begin{array}{l}1 \\
4 \\
5\end{array}$ & $\begin{array}{c}20,0 \\
80,0 \\
100,0\end{array}$ \\
\hline $\begin{array}{l}\text { 4. Lengua materna } \\
\text { (L1) }\end{array}$ & $\begin{array}{l}\text { Español } \\
\text { Griego } \\
\text { Total }\end{array}$ & $\begin{array}{l}1 \\
4 \\
5\end{array}$ & $\begin{array}{c}20,0 \\
80,0 \\
100,0\end{array}$ \\
\hline 5. Otras lenguas & $\begin{array}{l}\text { Griego e inglés } \\
\text { Inglés y francés } \\
\text { Inglés e italiano } \\
\text { Total }\end{array}$ & $\begin{array}{l}1 \\
2 \\
2 \\
5\end{array}$ & $\begin{array}{c}20,0 \\
40,0 \\
40,0 \\
100,0\end{array}$ \\
\hline $\begin{array}{l}\text { 6. Experiencia } \\
\text { docente }\end{array}$ & Más de 5 años & 5 & 100,0 \\
\hline $\begin{array}{l}\text { 7. Formación } \\
\text { continua }\end{array}$ & Sí. & 5 & 100,0 \\
\hline $\begin{array}{l}\text { 8. Importancia de } \\
\text { la interacción } \\
\text { oral en el aula }\end{array}$ & $\begin{array}{l}25 \% \text { de la clase } \\
50 \% \text { de la clase } \\
\text { Total }\end{array}$ & $\begin{array}{l}1 \\
4 \\
5 \\
\end{array}$ & $\begin{array}{c}20,0 \\
80,0 \\
100,0 \\
\end{array}$ \\
\hline $\begin{array}{l}\text { 9. La corrección es } \\
\text { una necesidad } \\
\text { para mis } \\
\text { alumnos } \\
\end{array}$ & Sí. & 5 & 100,0 \\
\hline $\begin{array}{l}\text { 10. Grado de } \\
\text { conciencia de las } \\
\text { técnicas de } \\
\text { corrección que } \\
\text { utilizo }\end{array}$ & $\begin{array}{l}\text { Sí. } \\
\text { A veces. } \\
\text { Total }\end{array}$ & $\begin{array}{l}4 \\
1 \\
5\end{array}$ & $\begin{array}{c}80,0 \\
20,0 \\
100,0\end{array}$ \\
\hline
\end{tabular}

Los profesores objeto de estudio son adultos con una edad media de entre $40 \mathrm{y}$ 50 años; todos profesionales con una extensa experiencia docente, e incluyen entre sus actividades la formación continua. Cuatro de ellos son hablantes no nativos (griegos) y uno es español; además de su lengua materna (L1) -griego o españoltodos ellos tienen el inglés como lengua extranjera y algunos, además, conocen una segunda lengua extranjera (francés, italiano y griego en el caso de la profesora española); de todos ellos, el $80 \%$ son mujeres, sólo hay un profesor varón.

En el marco de su perfil docente, nos ha interesado saber qué lugar ocupa la interacción oral en el aula y nos ha sorprendido de manera positiva el hecho de que un $80 \%$ de la muestra declara que esta habilidad lingüística ocupa un $50 \%$ de las actividades de la clase: este $80 \%$ coincide con los profesores que pertenecen al 
Centro de Idiomas Modernos de la Universidad de Atenas, mientras que el profesor que trabaja en la Universidad Kapodistríaca reduce este porcentaje al 25\%. Este resultado es de especial interés para nosotros porque nos sitúa ante un grupo docente en cuyas aulas la interacción oral presenta un protagonismo y, por ende, la actitud ante los errores y las técnicas de corrección encuentra un contexto propicio para su estudio. En este sentido, todos los profesores están de acuerdo en que la corrección en el uso del código es una necesidad para los estudiantes y un $80 \%$ declara ser consciente de las técnicas que utiliza en el aula para corregir o reparar los aspectos mejorables.

\section{ANÁLISIS Y DISCUSIÓN DE LOS DATOS}

Los datos, recogidos durante el curso académico 2012-13 han sido analizados mediante el paquete estadístico $\operatorname{SPSS}^{1}$ y se han realizado las pruebas pertinentes para llevar a cabo la descripción y comparación de los mismos. En esta investigación hemos querido ver si hay relación entre las creencias y actitudes del profesor y las de los alumnos, de esta manera, podremos constatar la consonancia o no entre las estrategias didácticas adoptadas por el profesor y las necesidades manifestadas por los alumnos, y es que una buena enseñanza debería acercar lo más posible estos dos aspectos ya que "una enseñanza adecuada de la lengua debe ocuparse de los procesos naturales, facilitar y agilizar el aprendizaje, en lugar de impedirlo" (Corder, 1981, 7).

La siguiente tabla presenta las variables que -resultado del cruce de los dos cuestionarios administrados- van a ser objeto de comparación y discusión:

Tabla 4. Variables comparadas

\begin{tabular}{|l|}
\hline 1. Consideración positiva del error \\
2. Consideración negativa del error \\
3. Error como un obstáculo para el aprendizaje \\
4. Error considerado negativo: intransigencia \\
5. Los errores contribuyen al aprendizaje: ayudan \\
6. La corrección: depende de la actividad \\
7. Quién corrige: el profesor \\
8. Quién corrige: también los alumnos a sus compañeros \\
9. Cuándo corregir: mientras se desarrolla la actividad \\
10. Cuándo corregir: después de terminarla actividad \\
11. Qué corregir: vocabulario \\
12. Qué corregir: pronunciación
\end{tabular}

${ }^{1}$ Queremos expresar nuestro agradecimiento a Pedro Cuesta, del Centro de Ayuda a la Investigación y la Docencia de la Universidad Complutense de Madrid, por su inestimable ayuda en el procesamiento estadístico de los datos. 


13. Qué corregir: gramática
14. Qué corregir: adecuación
15. Cómo corregir: corrección reparadora
16. Cómo corregir: indicación para autocorrección
17. Cómo corregir: después de una lista selectiva
18. Cómo corregir: autocorrección tras grabación

Tras emparejar las preguntas o variables de los respectivos cuestionarios, hemos aplicado la prueba t de Student para ver si las medias son similares o diferentes; las medias se han considerado significativas cuando la sig. es menor de $<0,05$, teniendo en cuenta y recordando que la puntuación de 1 significa estoy muy en desacuerdo y la puntuación de 5 estoy muy de acuerdo. A continuación agrupamos el análisis de los datos en dos apartados: por un lado, aquellos cuyas medias no hayan sido significativas y, por ende, la opinión de alumnos y profesores resulte bastante o muy coincidente; $y$, por otro lado, aquellas variables contrastadas en las que la media es significativa y reflejan aspectos en los que profesores y alumnos no están de acuerdo, indicio de que algo no funciona en el aula.

\subsection{Alumnos y profesores no están de acuerdo en sus respuestas}

En este apartado vamos a dar cuenta de aquellas variables en las que el análisis estadístico de los datos (prueba t) refleja que alumnos y profesores presentan un grado de desacuerdo significativo (sig.) en sus respuestas, tal y como aparece reflejado en la tabla 5:

Tabla 5. Comparación de variables alumnos vs. Profesores

Con valor significativo de desacuerdo

\begin{tabular}{|c|c|c|c|c|c|}
\hline & & $\mathbf{N}$ & Media & $\begin{array}{c}\text { Desviación } \\
\text { típica }\end{array}$ & Sig. \\
\hline $\begin{array}{l}\text { 1. Consideración } \\
\text { positiva del error }\end{array}$ & $\begin{array}{l}\text { Alumnos } \\
\text { Profesores }\end{array}$ & $\begin{array}{c}81 \\
5\end{array}$ & $\begin{array}{l}3,867 \\
2,900\end{array}$ & $\begin{array}{l}, 6732 \\
, 2236\end{array}$ & 0,002 \\
\hline $\begin{array}{l}\text { 2. Consideración } \\
\text { positiva del error: } \\
\text { ayuda a aprender }\end{array}$ & $\begin{array}{l}\text { Alumnos } \\
\text { Profesores }\end{array}$ & $\begin{array}{c}81 \\
5\end{array}$ & $\begin{array}{l}3,94 \\
4,80\end{array}$ & $\begin{array}{l}1,208 \\
, 447\end{array}$ & 0,118 \\
\hline $\begin{array}{l}\text { 3. Consideración del } \\
\text { error como un } \\
\text { obstáculo para el } \\
\text { aprendizaje }\end{array}$ & $\begin{array}{l}\text { Alumnos } \\
\text { Profesores }\end{array}$ & $\begin{array}{c}81 \\
5\end{array}$ & $\begin{array}{l}2,62 \\
1,60\end{array}$ & $\begin{array}{l}1,529 \\
, 894\end{array}$ & 0,146 \\
\hline $\begin{array}{l}\text { 4. Quién debe } \\
\text { corregir: el } \\
\text { profesor }\end{array}$ & $\begin{array}{l}\text { Alumnos } \\
\text { Profesores }\end{array}$ & $\begin{array}{c}81 \\
5\end{array}$ & $\begin{array}{l}3,91 \\
2,00\end{array}$ & $\begin{array}{l}1,027 \\
1,517\end{array}$ & 0,001 \\
\hline $\begin{array}{l}\text { 5. Quién puede } \\
\text { corregir también: }\end{array}$ & $\begin{array}{l}\text { Alumnos } \\
\text { Profesores }\end{array}$ & $\begin{array}{c}81 \\
5\end{array}$ & $\begin{array}{l}2,65 \\
4,00\end{array}$ & $\begin{array}{l}1,305 \\
1,225\end{array}$ & 0,027 \\
\hline
\end{tabular}




\begin{tabular}{|l|l|c|c|c|c|}
\hline el alumno & & & & & \\
\hline 6. Qué corregir: & Alumnos & 81 & 3,72 & 1,237 & 0,118 \\
pronunciación & Profesores & 5 & 4,60 &, 548 & \\
\hline
\end{tabular}

La consideración positiva del error, en términos generales y en el aspecto particular de que contribuye al aprendizaje y "resulta necesario e inevitable" (variables 1 y 2 de la tabla 5), presenta cierta contradicción en las valoraciones de alumnos y profesores: por un lado, los alumnos valoran de una forma más alta la consideración positiva del error (una puntuación de 3,8/5 frente a un 2,9/5 de los profesores; no obstante, en la variable que enfatiza el carácter positivo del error como parte del proceso de aprendizaje y ayuda en este sentido, son los profesores los que manifiestan casi un total acuerdo, frente a los alumnos, que están de acuerdo pero en un grado menor. Podemos colegir que ante la consideración positiva del error hay dos fuerzas contradictorias, aun prevaleciendo su carácter positivo: por un lado, la influencia de la literatura científica que defiende este aspecto desde finales de los años 60 y por otro, la experiencia del alumno, quien no deja de experimentar cierta frustración. De ahí que en la variable 3, los profesores, con larga experiencia y habituados a la formación continua, se manifiesten en desacuerdo con que los errores obstaculicen el aprendizaje, frente a los alumnos, que manifiestan sus dudas y afirman no estar ni de acuerdo ni en desacuerdo.

En las líneas que siguen vamos a relacionar y comentar las variables 4 y 5 , dedicadas a quién debe o puede corregir en el aula: ¿el profesor?, ¿los alumnos a sus compañeros?, ¿el profesor y los alumnos? Los profesores son vehementes en este aspecto, tanto ellos como los alumnos pueden y deben corregir; sin embargo, los alumnos consideran que el principal responsable de la corrección ha de ser el profesor. Cierto es que entre las estrategias docentes de los paradigmas comunicativos se defienden las actividades colaborativas, tanto reales como virtuales, en las que los alumnos son responsables de todo el proceso, incluida la corrección de lo dicho o escrito por sus compañeros; sin embargo, los compañeros no suelen tener el mismo grado de confianza en la autoridad de los alumnos frente a la que sí tiene para ellos el profesor.

Por último y en relación con la variable 6, que aborda uno de los aspectos sobre qué corregir -la pronunciación-, observamos que los profesores son categóricos en este sentido y se manifiestan muy de acuerdo, mientras que los alumnos se manifiestan ni de acuerdo ni en desacuerdo.

\subsection{Alumnos y profesores están de acuerdo en sus respuestas}

Las pruebas estadísticas realizadas muestran que hay un importante elenco de variables en las que profesores y alumnos manifiestan un grado de acuerdo muy similar -como se puede ver en la media-, de lo que se deduce que las estrategias 
docentes se encuentran en consonancia con las necesidades y preferencias de los alumnos:

Tabla 6. Comparación de variables alumnos vs. Profesores con valor significativo de acuerdo

\begin{tabular}{|c|c|c|c|}
\hline Variable & Informantes & $\mathbf{N}$ & Media \\
\hline $\begin{array}{l}\text { 1. Consideración negativa de los } \\
\text { errores }\end{array}$ & $\begin{array}{l}\text { Alumnos } \\
\text { Profesores }\end{array}$ & $\begin{array}{c}81 \\
5\end{array}$ & $\begin{array}{l}2,239 \\
2,533\end{array}$ \\
\hline $\begin{array}{l}\text { 2. Consideración negativa e intransigente } \\
\text { de los errores }\end{array}$ & $\begin{array}{l}\text { Alumnos } \\
\text { Profesores }\end{array}$ & $\begin{array}{c}81 \\
5\end{array}$ & $\begin{array}{l}1,57 \\
1,20\end{array}$ \\
\hline 3. Qué corregir: depende de la actividad & $\begin{array}{l}\text { Alumnos } \\
\text { Profesores }\end{array}$ & $\begin{array}{c}81 \\
5\end{array}$ & $\begin{array}{l}3,22 \\
3,40\end{array}$ \\
\hline 4. Cuándo corregir: mientras & $\begin{array}{l}\text { Alumnos } \\
\text { Profesores }\end{array}$ & $\begin{array}{c}81 \\
5\end{array}$ & $\begin{array}{l}3,25 \\
3,20\end{array}$ \\
\hline 5. Cuándo corregir: después & $\begin{array}{l}\text { Alumnos } \\
\text { Profesores }\end{array}$ & $\begin{array}{c}81 \\
5\end{array}$ & $\begin{array}{l}3,78 \\
3,60\end{array}$ \\
\hline 5. Qué corregir: vocabulario & $\begin{array}{l}\text { Alumnos } \\
\text { Profesores }\end{array}$ & $\begin{array}{c}81 \\
5\end{array}$ & $\begin{array}{l}4,11 \\
4,60\end{array}$ \\
\hline 6. Qué corregir: gramática & $\begin{array}{l}\text { Alumnos } \\
\text { Profesores }\end{array}$ & $\begin{array}{c}81 \\
5\end{array}$ & $\begin{array}{l}4,23 \\
4,60\end{array}$ \\
\hline 7. Qué corregir: adecuación & $\begin{array}{l}\text { Alumnos } \\
\text { Profesores }\end{array}$ & $\begin{array}{c}81 \\
5\end{array}$ & $\begin{array}{l}4,11 \\
4,40\end{array}$ \\
\hline 8. Cómo corregir: reparadora & $\begin{array}{l}\text { Alumnos } \\
\text { Profesores }\end{array}$ & $\begin{array}{c}81 \\
5\end{array}$ & $\begin{array}{l}4,22 \\
4,00\end{array}$ \\
\hline $\begin{array}{l}\text { 9. Cómo corregir: indicación para } \\
\text { autocorrección }\end{array}$ & $\begin{array}{l}\text { Alumnos } \\
\text { Profesores }\end{array}$ & $\begin{array}{c}81 \\
5\end{array}$ & $\begin{array}{l}3,62 \\
3,00\end{array}$ \\
\hline $\begin{array}{l}\text { 10. Cómo corregir: a posteriori con una } \\
\text { lista de errores frecuentes }\end{array}$ & $\begin{array}{l}\text { Alumnos } \\
\text { Profesores }\end{array}$ & $\begin{array}{c}81 \\
5\end{array}$ & $\begin{array}{l}3,52 \\
3,60\end{array}$ \\
\hline $\begin{array}{l}\text { 11. Cómo corregir: autocorrección con } \\
\text { grabación }\end{array}$ & $\begin{array}{l}\text { Alumnos } \\
\text { Profesores }\end{array}$ & $\begin{array}{c}81 \\
5\end{array}$ & $\begin{array}{l}2,38 \\
1,60\end{array}$ \\
\hline
\end{tabular}

\subsection{Comparación de variables intragrupos: A2, B1, B2 y C1}

Tal y como hemos defendido en las líneas precedentes, a nuestro modo de ver, resulta fundamental que profesor y alumnos consensuen las estrategias didácticas en relación con la corrección de los errores y que esto se refleje en el aula, por ello, hemos creído importante contrastar las creencias de alumnos y profesor por grupo. Procedemos con las mismas pruebas estadísticas ya mencionadas y solo damos cuenta de aquellas variables en las que las respuestas de alumnos y profesor difieren de manera significativa (tal y como expresa la columna sig.): 
Tabla 7. Nivel a2

\begin{tabular}{|c|c|c|c|}
\hline & N & Media & Sig. \\
\hline & A: 21 & 4,262 & \\
1. Consideración positiva del error & P: 1 & 3,000 & 0,33 \\
\hline & A: 21 & 3,95 & \\
2. Quién corrige: el profesor & P: 1 & 1,00 &, 005 \\
\hline & A: 21 & 4,52 & \\
3. Cuándo corregir: después & P: 1 & 2,00 & 0,002 \\
\hline
\end{tabular}

En el nivel A2, alumnos y profesor manifiestan un desacuerdo en relación con tres de las variables: 1. Consideración positiva del error: los alumnos consideran que los errores son positivos y forman parte del aprendizaje, frente al profesor, quien no está de acuerdo ni en desacuerdo; 2. Quien corrige es el profesor: en este aspecto, los alumnos son vehementes al considerar que es el profesor quien debe corregir; y 3. Corregir después de la intervención: los alumnos prefieren ser corregidos tras su intervención, en ningún caso les gusta que les interrumpan, mientras que el profesor se manifiesta en desacuerdo. En todas las demás variables presentan acuerdo en algún grado.

En el nivel B1 no se ha constatado ninguna discrepancia entre alumnos y profesor.

Tabla 8. Nivel B2

\begin{tabular}{|c|c|c|c|}
\hline & N & Media & Sig. \\
\hline & A: 21 & 4,025 & 0,33 \\
1. Consideración positiva del error & P: 1 & 3,000 & \\
\hline
\end{tabular}

En el nivel B2, alumnos y profesor solo han manifestado su discrepancia y, no en un grado muy alto dentro de los parámetros significativos, en relación con la consideración de los errores: los alumnos se manifiestan más favorables a ver los errores como un fenómeno positivo frente al profesor que, como viene siendo habitual, mantiene una postura neutra.

Tabla 9. Nivel C1a

\begin{tabular}{|l|c|c|c|}
\hline & N & Media & Sig. \\
\hline & A: 21 & 4,13 &, 010 \\
1. Consideración negativa del error & P: 1 & 1,00 & \\
\hline & A: 21 & 4,50 &, 000 \\
2. Quién corrige: el profesor & P: 1 & 1,00 & \\
\hline & A: 21 & 2,63 &, 021 \\
3. Cómo corregir: autocorrección con & P: 1 & 100 & \\
grabación & & & \\
\hline
\end{tabular}


En el nivel C1 (grupo a $=8$ alumnos del Centro de Idiomas Modernos de la Universidad de Atenas), alumnos y profesor discrepan en tres variables: 1 . Consideración negativa del error: los alumnos están muy de acuerdo en considerar los errores como algo negativo, hecho que contrasta totalmente con los alumnos de los grupos A2 y B2, mientras que el profesor está en total desacuerdo con que los errores sean considerados como un fenómeno negativo.

Tabla 10. Nivel C1b

\begin{tabular}{|l|c|c|c|}
\hline & N & Media & Sig. \\
\hline & A: 21 & 4,33 &, 002 \\
1. Quién corrige: el profesor & P: 1 & 1,00 & \\
\hline & A: 21 & 3,33 &, 032 \\
2. Qué corregir: depende de la & P: 1 & 5,00 & \\
actividad & & & \\
\hline & A: 21 & 4,50 &, 004 \\
3. Cómo corregir: reparadora & P: 1 & 2,00 & \\
\hline & A: 21 & 4,08 &, 028 \\
4. Cómo corregir: indicación para & P: 1 & 2,00 & \\
autocorrección & & & \\
\hline
\end{tabular}

En el nivel C1 (grupo b = 13 alumnos de la Universidad Kapodistríaca de Atenas), el resultado es de cuatro variables con discrepancia entre alumnos y profesor: los alumnos consideran que quien debe corregir es siempre el profesor, que el tipo de actividad que se esté realizando no influye totalmente en la corrección y, por último, manifiestan su preferencia por el tipo de corrección reparadora y por la indicación para autocorrección.

\section{CONCLUSIONES}

Este estudio se cierra con unas breves conclusiones que pasamos a comentar y que presentamos con la cautela propia de la investigación científica.

En términos generales y en relación con las variables que hemos establecido para estudiar el consenso entre las creencias o teorías implícitas de profesores y alumnos respecto de los errores y las técnicas de corrección en la interacción oral, el análisis de los datos constata que hay un mayor acuerdo (en 12 variables) que desacuerdo (en 6 variables), lo que nos permite afirmar que hay un grado alto de consistencia entre las creencias y preferencias de los alumnos y la actuación del profesor en el aula.

En cuanto a la consideración de los intragrupos A2, B1, B2 y C1, es decir, la comparación de cada grupo de alumnos y su respectivo profesor, se observa que el mayor número de discrepancias y, por lo tanto, de falta de acuerdo entre las preferencias de los alumnos y las técnicas docentes, se dan en el nivel inicial (A2) 
y en el avanzado (C1). Si comparamos el pensamiento del alumno en estos dos estadios del aprendizaje, resulta llamativo, aunque en consonancia con la lógica del proceso de aprendizaje, que en el nivel A2 los alumnos consideran el error como algo positivo, mientras que en el nivel $\mathrm{C} 1$ predomina la consideración negativa: en los primeros estadios, los errores son vistos como signo de desarrollo del proceso, sin embargo, en un nivel más avanzado son considerados objeto de frustración en el dominio de la lengua y en el desarrollo de su competencia comunicativa. Por lo demás, y solo en relación con las variables en las que discrepan profesor y alumnos, destacan dos aspectos: por una parte, los alumnos consideran que deben ser corregidos exclusivamente por el profesor tanto en el nivel inicial como en el avanzado; $\mathrm{y}$, por otra, en el nivel $\mathrm{C} 1$ hay una preferencia por las correcciones reparadoras.

\section{BIBLIOGRAFÍA}

ABELSON, R. (1979): "Differences between beliefs and knowledge systems", Cognitive Science, 3 (4), 355-366.

ARNOLD, J. (2006): "Los factores afectivos en el aprendizaje del español como lengua extranjera", Études de Linguistique Appliquée, 139. http://cvc.cervantes.es/ensenanza/biblioteca_ele/antologia_didactica/claves/arno ld.htm [Consulta: 20 de octubre de 2013].

CHAPARRO, M. (2008): Estudio descriptivo de la actitud del hablante no nativo de español ante la corrección de errores en la interacción oral en los Niveles Intermedio 1, Cursos Cuarto y Quinto de la Escuela Oficial de Idiomas "Jesús Maestro" de Madrid, Trabajo de Investigación inédito. Madrid: Universidad Complutense.

CONSEJO DE EUROPA (2002): Marco común europeo de referencia para las lenguas: aprendizaje, enseñanza, evaluación, Madrid, Ministerio de Educación, Cultura y Deporte - Instituto Cervantes - Editorial Anaya [MCER].

CORDER, S.P. (1967): "The Significance of learner's errors", International Review of Applied Linguistics, 5, 161-170, en J. MUÑOZ LICERAS (Ed.) (1991): La Adquisición de las lenguas Extranjeras, Madrid, Visor, 31-40).

DÍAZ MARTÍNEZ, J. (1998): Afectividad y tratamiento del error en la clase de segundas lenguas: estudio introspectivo de un diario, Barcelona, Universitat de Barcelona.

FERNÁNDEZ DE AMO, M. (2008): "Una visión afectiva del error oral en el aula de inglés", Revista Digital "Práctica Docente", 9, 39-48.

GROULT BOIS, N. (2005): 'Para mí, aprender una lengua significa...' representaciones de alumnos universitarios mexicanos acerca de lenguas extranjeras", ELIA 6, 93-114.

HORWITZ, E. K. (1987): "Surveying students' beliefs about language learning", en A. WENDEN Y J. RUBIN (Eds.): Learner's Strategies in Language Learning, Englewood Cliffs, NJ, Prentice-Hall, 119-129. 
JOETZE, S. (2007): Oral Error Treatment in the Second Language Classroom, Alemania, Grin Verlag.

LARSEN FREEMAN, D. Y LONG, M. H. (1994): Introducción al estudio de la adquisición de segundas lenguas, Madrid, Gredos.

MARTÍNEZ AGUDO, J. de D. (Ed.) (2010): Errors in the Second Language Classroom: Corrective Feefback, Málaga, Ediciones Aljibe.

MEKASSIMOVE, S. (2009): "Las creencias de los alumnos de la secundaria gabonesa acerca de su proceso de aprendizaje del español/LE", MarcoELE, 9, $1-17$.

NUNAN, D. (1992): Research Methods in Language Learning, Cambridge, Cambridge University Press.

PUCHTA, H. (2000): "Creación de una cultura de aprendizaje a la que el alumno quiera pertenecer: la aplicación de la programación neuro-lingüística en la enseñanza de idiomas", en J. ARNOLD (Ed.) (2000): La dimensión afectiva en el aprendizaje de idiomas, Madrid, Cambridge University Press, 263-275.

PAJARES, M. F. (1992): "Teacher's beliefs and educational research: cleaning up a messy construct", Review of Educational Research, 62 (3), 307-332.

PICÓ, E., VERDÉS, G Y VILAGRASA, A. (1988): "Las teorías implícitas, estrategias y preferencias de los estudiantes de E/SL en relación a su nivel de toma de riesgo. Algunas correlaciones e implicaciones pedagógicas". ASELE, Actas $I$.

RAMOS MÉNDEZ, C. (2007): El pensamiento de los aprendientes en torno a cómo se aprende una lengua: dimensiones individuales y culturales. ASELE, Colección Monografías $n^{\circ} 10$.

RICHARDS J.C, Y LOCKHART, C. (1998): Estrategias de reflexión sobre la enseñanza de idiomas. Cambridge, Cambridge University Press.

RONCEL VEGA, V. M. (2008): "Autoconcepto, motivación y ansiedad en el aula de idiomas", MarcoELE, 7, 1-19.

SERRA, R. (1997): "Las representaciones de los profesores sobre la enseñanzaaprendizaje de lenguas. Estado de la cuestión desde la perspectiva institucional del currículum de lenguas", en M. L. VILLANUEVA E I. NAVARRO (Eds.), Los estilos de aprendizaje de lenguas, Castelló, Collección Summa, 155-175.

VAN DIJK, T. (1999): Ideología: una aproximación multidisciplinaria, Barcelona, GEDISA.

WILLIAMS, M, Y BURDEN, R.L. (1999): Psicología para profesores de idiomas, Madrid, Edinumen.

YIN, R. (1984): Case study research: Design and methods, Beverly Hills, SAGE. 